\title{
Orientation Spectral Resolution Coding for Pattern Recognition
}

\author{
Shaikh Afroz Fatima Muneeruddin \\ Research Scholar, Shri Jagdishprasad Jhabarmal Tibrewala University, Rajasthan, India. \\ afrozphatima@gmail.com
}

\begin{abstract}
In the approach of pattern recognition, feature descriptions are of greater importance. Features are represented in spatial domain and transformed domain. Wherein, spatial domain features are of lower representation, transformed domains are finer and more informative. In the transformed domain representation, features are represented using spectral coding using advanced transformation technique such as wavelet transformation. However, the feature extraction approach considers the band coefficients; the orientation variation is not considered. In this paper towards inherent orientation variation among each spectral band is derived, and the approach of orientation filtration is made for effective feature representation. The obtained result illustrates an improvement in the recognition accuracy, in comparison to conventional retrieval system.
\end{abstract}

Index Term: Pattern recognition, orientation spectral resolution, wavelet transformation, spectral features

\section{Introduction}

Pattern recognition has remained an area of research from past a decade. With the emergence of new technologies, information sharing has become more effective in distributed domain. For the information's exchange from one location to other, or to retrieve data from a large information data set, automated retrieval systems are in greater demands. In various means of applications, information sharing, retrieval, and processing is a common task. These tasks are limited with their usage and resources availability. In almost all processing system, obtained information's are very much dependent on the accuracy of data given to process. Generally the processing data are taken as direct input or as retrieved information from a large dataset. Wherein direct inputs from user are constraint to information knowledge, it is always used as a querying process. Based on the query passed, mining algorithms were executed to retrieve the nearest Match information's from a volumetric data set. The accuracy of such process depends on the retrieval algorithm and the representative features. These algorithms, are developed, modified and evaluated to improve the retrieval system performances. Towards the application of wavelet transformation for feature description in CBIR system, various approaches were developed in past. In [1] for the purpose of efficiently and effectively retrieving the desired images from a large image database, the development of a user-friendly image retrieval system is developed. In this paper, a content based image retrieval method based on the discrete wavelet transform (DWT) based on the superiority in multi resolution analysis and spatial-frequency localization. An interactive user interface such that the users can adjust the weights for each wavelet feature according to their expectations is developed. The effect of wavelet feature selection is evaluated. In [2] a novel CBIR method is proposed by exploit the wavelets which represent the visual feature. Haar and D4 wavelet to decompose color images into multilevel scale and wavelet coefficients, with which image feature extraction and similarity match by means of F-norm theory is carried out. A progressive image retrieval strategy to achieve flexible CBIR is also proposed. In [3] a discrete wavelet transform with texture for content based image retrieval is proposed. This method uses a 2-D Discrete Wavelet Transform for reducing the Dimensions of test image and trained images. Further gray level co-occurrence matrix is applied for all test and trained images of LL components of level 2 decomposed images for extract the texture feature of the images. In [4] a wavelet-based salient point extraction algorithm is proposed. The color and texture information in the locations given by these points provides significantly improved results in terms of retrieval accuracy, computational complexity and storage space of feature vectors as compared to the global feature approaches. A performance comparison is done on various image transforms like Wavelet transform, Fourier transform, Haar transform, Walsh-Hadamard transform and discrete cosine transform using a fuzzy similarity measure is presented in $[5,6]$. It is seen that according to retrieval performance Wavelet transform gives the best result among the other mentioned transforms. It has higher recall and precision values and higher crossover point. In [7] an application system which implements a retrieval method which combines color and texture feature for retrieval of images from large image databases is proposed. In this the process retrieve images by using techniques like Wavelet decomposition, Color correlogram, Median Cut algorithm, and Color Mean. For testing the final feature vectors of an input image with the database images a SVM (Support Vector Machine) classifier is used. A similar modeling is observed in [8], where an image retrieval approach based on color, texture feature is developed. Wavelet based approach is proposed to achieve the objective of feature extraction and a classifier using K-nearest neighbors Algorithm (KNN) is developed to perform the pattern 
recognition approach. These approaches improved the retrieval performances in various applications. In [9] a Wavelet Transform based analysis method for Face Recognition is proposed. The choice of the Wavelet transform in this setting is motivated by its insensitivity to large variation in light direction, face pose, and facial expression. In a similar approach in [10] the illumination conditions that effect the automatic face recognition process is focused. In this work, a multi-resolution feature extraction algorithm for face recognition is proposed based on two-dimensional discrete wavelet transform (2D-DWT), which efficiently exploits the local spatial variations in a face image. Wavelet coefficients corresponding to each local region residing inside those horizontal bands are selected as features. In the selection of the dominant coefficients, a threshold criterion is proposed, which drastically reduces the feature dimension.

\section{Pattern Recognition}

The image recovery systems are determined with generally the content features of the image named as shape or texture, color recognition. In this paper information of color-based is taken as the referencing index and using DWT method the spectral variation can be calculated. In input image information we can extract the frequency resolution information very efficiently using DWT method. Where the resolution information was passed as additional information for recovery in past, computational complexity increases because increasing the feature count result. To achieve the objective of image retrieval, the operation is performed in two stages,

(i) training and

(ii) testing.

A Basic architecture for such a system is shown in figure 1 .



Figure 1: Fundamental architecture of a content based image retrieval system

In training and testing the samples are pre-processed for resizing, filtration and data precision. The preprocessing sample is further processes for feature extraction. In this stage image features are extracted namely shape or texture, color recognition. In this paper information of color-based is taken as the referencing index and using DWT method the spectral variation can be calculated. In input image information we can extract the frequency resolution information very efficiently using DWT method. Where the resolution information was passed as additional information for recovery in past, computational complexity increases because increasing the feature count result.

\section{Spectral Resolution Coding}

In image and signal processing DWT place an important role, particularly in spectral-resolution representation. In image processing system, it is very difficult to get the intensity of gray-level image pixels of information directly. But using Spectral-resolution we can get the information of image easily. The 2D-DWT can divide an image into four different sub-bands. Those sub-bands contain three detailed component sub-bands and one average sub-band. Those three detail sub-bands components can represent different features for an image. Wavelets $\psi_{\mathrm{a}, \mathrm{b}}(\mathrm{x})$ are functions generated from mother wavelet $\psi$ by dilations and translations

$$
\psi_{\mathrm{a}, \mathrm{b}}(\mathrm{x})=|\mathrm{a}|^{-1 / 2} \psi\left(\frac{x-b}{a}\right)
$$

In wavelet transform function $\mathrm{f}$ represents the wavelets superposition. Wavelet can be divided using weighted coefficients, as an integral over a range a and b of $\psi(\mathrm{x})$. In a spectral resolution analysis, a scaling function $\phi(\mathrm{x})$ is employed to process the Spectral-resolution. The wavelet can be divided into a $\mathrm{m}, \mathrm{n}(\mathrm{f})$ called as approximate coefficients of $\mathrm{a}_{\mathrm{m}-1,1}$ and $\mathrm{c}_{\mathrm{m}, \mathrm{n}}(\mathrm{f})$ termed as detail coefficients of $\mathrm{a}_{\mathrm{m}-1,1}$ using a low-pass and a highpass filter in cascade. The 2D decomposition is out by the combination of two 1D decomposition of WT. The

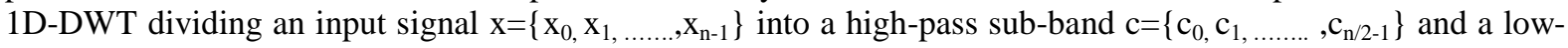
pass sub-band $a=\left\{a_{0}, a_{1}, \ldots \ldots . ., a_{n} / 2-1\right\}$. Those can be represented as, 


$$
\begin{gathered}
A_{n}=\sum_{k} h_{2 n-k} x_{k} \\
C_{n}=\sum_{k} g_{2 n-k} x_{k}
\end{gathered}
$$

Where $g_{n}$ and $h_{n}$ are the high-pass and low-pass filter coefficients respectively.

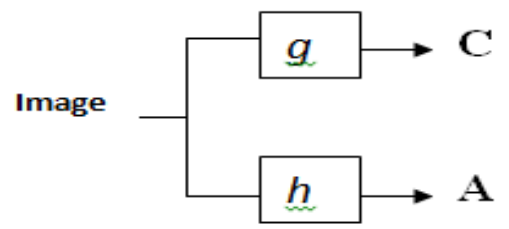

Figure 2: 1-D DWT Decomposition

Figure 2 shows the recognition of a 1D-DWT filter banks for dividing of original into detail sub-band coefficients respectively. The original image can be extracts the filter bank into two components one is high components and another is low components. In a 2D-DWT can be comes from the two 1D-DWT of rows and columns operations. Using 1D-DWT firstly done the row operation, on high -pass sub-band(H) and one lowpass sub-band (L) as shown in figure 3. The 1D-DWT image is again transformed column operation is done to get the four sub-bands by using another 1D-DWT. Figure 3 shows the 2D-DWT filter bank decomposition operation. The LL sub-band represents the average component and LH, HL and HH sub-bands represents the detail components.



Figure 3: Dimensional DWT Decomposition

There are many ways to divide a signal into various sub-bands in wavelet transforms. These contain octave-band decomposition, wavelet-packet or adaptive decomposition and uniform decomposition. Octaveband decomposition is mostly used.

\section{Orientation Spectral Resolution Coding}

An integrated model for selective coding in image coding is developed. The block diagram represented below in figure 4 gives the pictorial description of the proposed orientation filtering based image coding scheme.

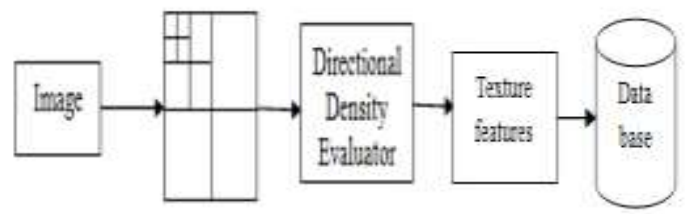

Figure 4: Proposed Orientation Spectral resolution coding for image compression

Using directional density evaluator we can extract the 2D image features easily, which is most preferable in image analysis. Using DDE we can get the total number of sub bands' coefficients is the same as that of the original image is called maximally decimated (MD) and reconstructed the original image without any error is called perfect reconstruction (PR). Using tree structure three levels of two-band system can be implemented in DDE. By using ploy-phase filter implemented each and every level, which construct the structure very efficient computationally as well as extremely. DDE (directional density evaluator) divides the frequency space into wedge-shaped as shown in fig.5. 


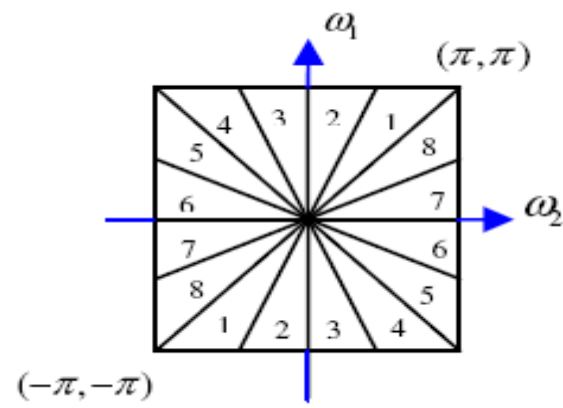

Figure 5: Directional density evaluator frequency partitioning using 8 directions

In this 8 directions were used, where sub-bands directional of $1,2,3$, and 4 indicates the directions between $-45^{\circ}$ and $+45^{\circ}$ (horizontal directions) and 5,6,7 and 8 indicates the directions between $45^{\circ}$ and $135^{\circ}$ (vertical directions). To get the objective selection of coefficient for embedding, the output of WT are processed with directional density evaluator and each band of 8-orientation is done. Rather than taking whole bands taking only lower coefficient from each band and select the embedding locations. To realize the DDE, quincunx filter banks are used. The filter arrangement is shown in fig.6.



Figure 6: Quincunx filter bank architecture

QFB (Quincunx filter bank) is applied at the $1^{\text {st }}$ level. The sampling matrix of quincunx id denoted by, $Q=$ $\left[\begin{array}{cc}1 & -1 \\ 1 & 1\end{array}\right]$. The filtration is further divided by using two quincunx filter banks for the o/p's $\mathrm{y}_{0}$ and $\mathrm{y}_{1}$. As a result four directions will get the four outputs. These four directional outputs are represented by $\mathrm{y}_{00}, \mathrm{y}_{01}, \mathrm{y}_{10}$ and $\mathrm{y}_{11}$. The quincunx filter banks of higher level results are shown in conjunction with re-sampling metrics. The resampling coefficients used per horizontal and vertical directions as shown in below,

$$
R_{h}=\left[\begin{array}{ll}
1 & 1 \\
0 & 1
\end{array}\right] \text { and } R_{v}=\left[\begin{array}{cc}
1 & 0 \\
-1 & 1
\end{array}\right]
$$

Each and every band apply sampling matrix and done the orientations, 8-orintations in results as $\mathrm{y}_{0}$ to $\mathrm{y}_{8}$. For each of this directional band a normalized magnitude value is computed defined by,

$$
N=\left\|y_{i, j}\right\|^{2}, \text { for } \mathrm{i}, \mathrm{j} \in[0,1]
$$

The band density having higher pixel density in such directions is kept unmodified and band having lower normalized values are discarded from coding. As these are made on the directional band density hence the image are least effected on the orientation variations. For this purpose the normalized magnitudes of all directional bands are compared with a normalized threshold value given in (2). The Coefficients satisfying the thresholding based criterion $N=|| y_{i, j}|\quad|^{2}>t h$ are only used for coding, the threshold $(t h)$, is defined by,

$$
t h=\frac{1}{3}\left[\left(\sum_{i=1}^{m} \cdot \sum_{j=1}^{n} \cdot x_{i, j}\right)\right] \frac{1}{m x n}
$$

The process of thresholding results in selection of effective oriented coefficients in each band and finally the obtained normalized magnitudes in all directional bands are passed to code for compression. Wherein the selective process reduces the coefficients having similarity in orientation, the reflecting visualization is not degraded due to psycho visual redundancy. Taking these orientations as feature vectors a recognition system is evaluated. 


\section{Experimental Results}

Columbia Object Image Library (COIL-100) is a database of color images of 100 objects. The objects were placed on a motorized turntable against a black background. The turntable was rotated through 360 degrees to vary object pose with respect to a fixed color camera. Images of the objects were taken at pose intervals of 5 degrees. This corresponds to 72 poses per object. The images were size normalized. COIL-100 is available online via ftp. i 1 Introduction We have constructed a database of 7,200 color images of 100 objects (72 images per object). The objects have a wide variety of complex geometric and reflectance characteristics (see figure 7. The database, called Columbia Object Image Library (COIL-100), was used in a real-time 100 object recognition.

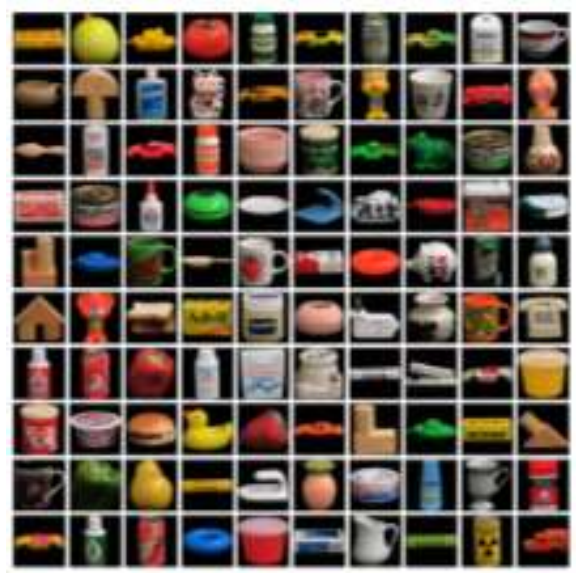

Figure 7: Database samples coil-100 Dataset

The proposed system is developed over Matlab tool, and tested over coil dataset. To evaluate the performance of the proposed approach a comparative analysis of conventional based feature dimension reduction is compared with the proposed orientation spectral resolution coding is carried out. The observed parameters of evaluation are outlined below,

$$
\text { Accuracy }=\frac{T P+T N}{T P+T N+F P+F N}
$$

Along with accuracy, to show the enhancement of propose approach and also to compare the proposed approach with earlier approaches, few more metrics such as sensitivity, specificity, Recall, precision and F-measure were evaluated with following mathematic expressions.

Sensitivity measures the proportion of positives that are correctly identified as such.

$$
\text { Sensitivity }=\frac{T P}{T P+F N}
$$

Specificity measures the proportion of negatives that are correctly identified as such.

$$
\text { Specificity }=\frac{T N}{T N+F P}
$$

Precision is the fraction of identified instances that are correct, while recall is the fraction of correct instances that are identified.

$$
\begin{aligned}
& \text { Recall }=\frac{T P}{T P+F N} \\
& \text { Precision }=\frac{T P}{T P+F P}
\end{aligned}
$$

F-measure or balanced F-score is a measure that combines precision and recall is the harmonic mean of precision and recall.

$$
F-\text { measure }=\frac{\text { 2.Recall } \text { Precision }}{\text { Recall }+ \text { Precision }}
$$


To evaluate the process of orientation Spectral resolution coding feature processing a test sample with different orientation is passed to the developed system. The obtained observations for the developed system is as illustrated below,

a) Sample I: Sample with $0^{0}$ orientation

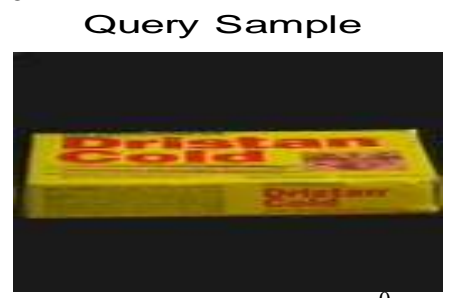

Figure 8: Original Test sample at $0^{0}$ orientations

A selected test sample for the recognition process is shown in figure 8. This sample is passed for feature extraction using Gray-Level Co-Occurrence Matrix (GLCM) based feature extraction and orientation Spectral resolution coding (OSRC) based feature extraction. The classification and retrieval for the given query is as shown below.


(a)

(b)



Figure 9: Top 4 classified sample using (a) OSRC (b) GLCM

The top 4 classified sample from the trained data base for the proposed OSRC and GLCM feature extraction is shown in figure 9 (a) and (b) respectively for the given test sample.

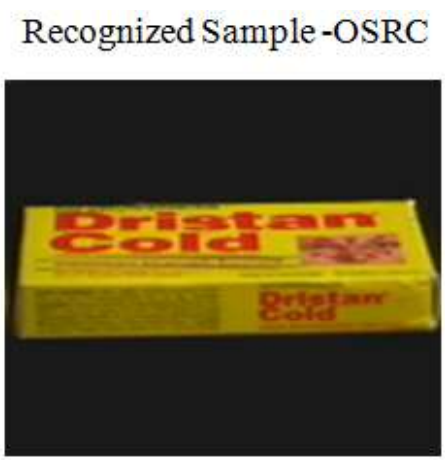

(a)

\section{Recognized Sample- GLCM}

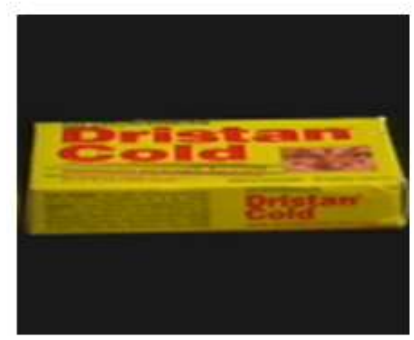

(b)

Figure 10: Final retrieved sample (a) Using OSRC (b) GLCM 
The top retrieved sample from the data base after classification for the two methods is shown in figure 10 (a) and (b) respectively. It is observed that the retrieval accuracy of the tow method is highest as the samples are with no orientation effect. This test sample is then evaluated over different orientation and the obtained results are as illustrated below;

\section{b) Sample II: Sample with $15^{0}$ orientation}

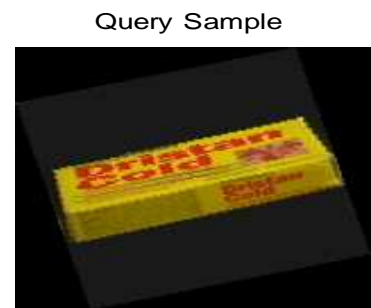

Figure 11: Test sample at orientation of $15^{0}$

The test sample is oriented by $15^{\circ}$ orientations, and passed as a test sample to the developed system. The features are extracted using conventional GLCM features and OSRC features. Using theses extracted features, recognition process is carried out. The obtained results of the classified observations are illustrated in figure 12 .




(a)

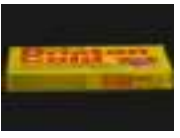

(b)

Figure 12: Top 4 classified sample using (a) OSRC (b) GLCM at $15^{\circ}$ orientations

When applied with orientation it is observed that the features extracted from the OSRC, in addition to the shape information, depth feature is considered, this feature reveals the orientation information, and hence these features variations are more contributive to the recognition process. However this variation is retained in GLCM as no orientation effect is recorded in such approach. Hence the classification process leads to misclassification process.

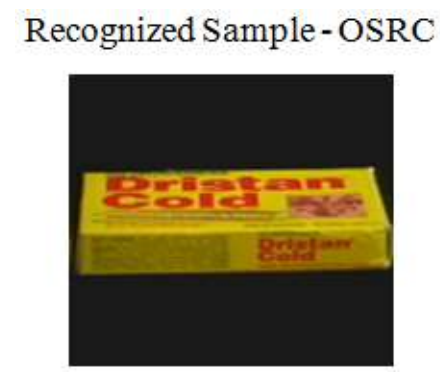

(a)

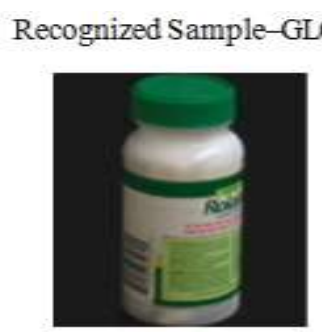

(b)

Figure 13: Final retrieved sample (a) Using OSRC (b) GLCM at $15^{\circ}$ orientations 
c) Sample III: Sample with $\mathbf{4 5}^{\circ}$ orientation

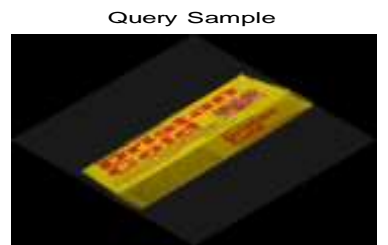

Figure 14: Test sample at $45^{\circ}$ orientations
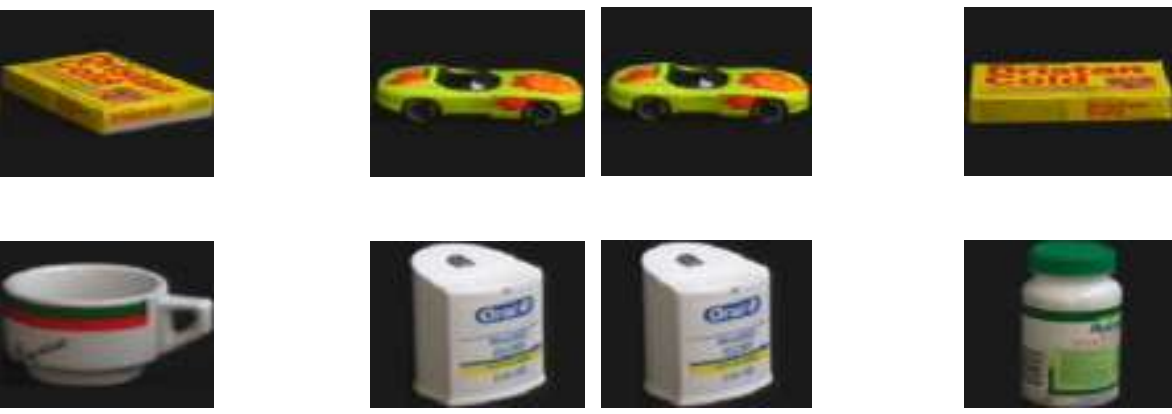

(a)

(b)

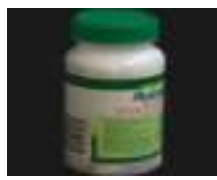

Figure 14: Top 4 classified sample using (a) OSRC (b) GLCMat $45^{\circ}$ orientations

Recognized Sample-OSRC



(a)

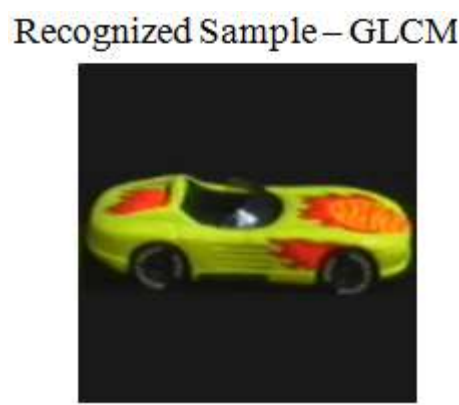

(b)

Figure 15: Final retrieved sample (a) Using OSRC (b) GLCM at $45^{\circ}$ orientations

The test result for the given test sample. Illustrates a retrieval accuracy of 9/10, for the given test sample. To evaluate the performance of proposed approach, one more numerical parameter, True Positive Factor (TPF) and False Positive Factor (FPF) used. TPF is defined as the ratio of the number of truly classified samples over the total available data samples. It finds the number of query samples classified truly according to their labels. FPF is defined as number of falsely classified samples over the total available data samples. FPF finds the number of samples that are classified wrongly. Mathematically, TPF and FPF can be expressed as

$$
\begin{aligned}
T P F & =\frac{\text { No.oftruely recognized samples }}{\text { total available samples in dataset }} \\
F P F & =\frac{\text { No.offalsely recognized samples }}{\text { tot al available samples in dataset }}
\end{aligned}
$$


For the proposed work,

Total samples available in dataset $=100$.

Samples taken for testing $=30$.

Number of truly classified samples $=29$.

Number of falsely classified samples $=1$.

The table shown below describes the TPF and FPF performance of the proposed and earlier approaches.

Table.1 TPF and FPF performance comparison

\begin{tabular}{|c|c|c|c|c|}
\hline \multirow{2}{*}{ Total Samples } & \multicolumn{2}{|c|}{ TPF } & \multicolumn{2}{c|}{ FPF } \\
\cline { 2 - 5 } & GLMC & OSRC & GLMC & OSRC \\
\hline 100 & $26 / 100=0.2673$ & $28 / 100=0.2878$ & $4 / 100=0.040$ & $2 / 100=0.020$ \\
\hline
\end{tabular}

A generalized plot for the above observation was created and shown in fig. 16. The lot was drawn between FPF and TPF, also can be called ROC plot.

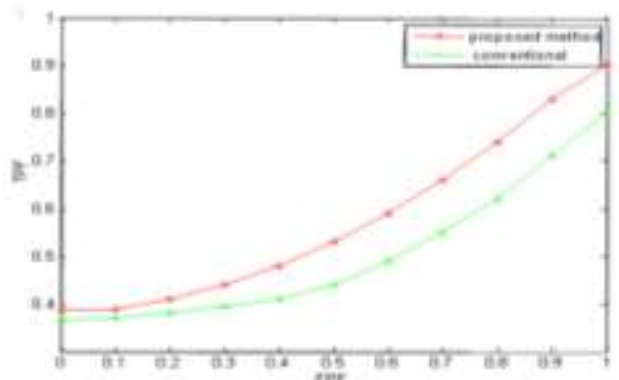

Fig.16: ROC analysis

The above figure represents the ROC curve for proposed approach and earlier approaches. The proposed approach was carried out under various tests by varying number of testing samples and FPF and the obtained results are shown in the fig.17.Form the above figure, as the FPF is increasing, the TPF is also increasing. The increment in the FPF shows the probability of getting false results. The increment if TPF denotes the probability of getting true results.

\section{Conclusion}

A new approach to feature representation, based on spectral orientation, in the spectral band decomposition is illustrated. The approach results in extraction of feature elements which are more effective in descriptive representation, as compared to conventional wavelet based approach. The coding approaches, extracts the non redundant feature coefficients as the descriptive elements, and hence are more finer in representation. This results in improvement to accuracy in pattern recognition and hence lead to a more accurate recognition system in image retrieval system.

\section{References}

[1]. Te-Wei Chiang, Tien-Wei Tsai, "Content-Based Image Retrieval via the Multiresolution Wavelet Features of Interest”, Journal of Information Technology and Applications Vol. 1 No. 3, December, 2006.

[2]. Y. M. Latha, B.C.Jinaga, V.S.K.Reddy, "Content Based Color Image Retrieval via Wavelet Transforms", International Journal of Computer Science and Network Security, VOL.7 No.12, December 2007.

[3]. Mohan kumar, Madhavan J, "Content Based Image Retrieval Using 2-D Discrete Wavelet with Texture Feature with Different Classifiers", IOSR Journal of Electronics and Communication Engineering, Vol. 9, Issue 2, 2014.

[4]. Q. Tian, N. Sebe, M.S. Lew, E. Loupias, T. S. Huang, “Content-Based Image Retrieval Using Wavelet-based Salient Points", SPIE Photonics West, Electronic Imaging 2001, Storage and Retrieval for Media Databases, January, 2001.

[5]. AlinaBanerjeea, AmbarDuttab, "Performance Comparison of Cosine, Haar, Walsh-Hadamard, Fourier and Wavelet Transform for shape based image retrieval using Fuzzy Similarity Measure", International Conference on Computational Intelligence: Modeling Techniques and Applications (CIMTA) Procedia Technology, Elsevier, 2013.

[6]. OmprakashYadav, VaishaliSuryawanshi, "Comparative Evaluation of Transform Based CBIR Using Different Wavelets and Two Different Feature Extraction Methods", International Journal of Computer Science and Information Technologies, Vol. 5 (5), 2014.

[7]. E.Sirisha, P.Sanoop Kumar, P.Hema Vishnu, Y. Srinivas, "Image Retrieval using wavelet decomposition, color correlogram and color mean", International Journal of Advanced Research in Computer Science and Software Engineering, Vol.3, Issue 9, Sept. 2013.

[8]. Ms. Pragati Ashok Deole, Prof. RushiLongadge, "Content Based Image Retrieval using Color Feature Extraction with KNN Classification”, IJCSMC, Vol. 3, Issue. 5, May 2014.

[9]. SangeetaKakarwal, RatnadeepDeshmukh, "Wavelet Transform based Feature Extraction for Face Recognition", International Journal of Computer Science and Application Issue, 2010. 
[10]. M.Karthika, K.Shanmugapriya, Dr.S.Valarmathy, M.Arunkumar, "Wavelet Based Face Recognition for Low Quality Images", International Journal of Advanced Research in Electrical, Electronics and Instrumentation Engineering Vol. 2, Issue 1, January 2013.

[11]. Ali Mohsin Al-juboori, Wei Bu, Xiangqian Wu, and Qiushi Zhao, “Palm Vein Verification Using Multiple Features and Isometric Projection”, International Journal of Signal Processing, Image Processing and Pattern Recognition Vol.7, No.1, 2014.

[12]. SavitaBorole, Prof. S. D. Sapkal, "Extraction of Dual Tree Complex Wavelet Feature for IRIS Recognition", International Journal of Advanced Research in Computer and Communication Engineering Volume 2, Issue 7, July 2013.

[13]. S. M. Rajbhoj, P. B. Mane, "Haar Wavelet Approach of Iris Texture Extraction for Personal Recognition", International Journal of Innovative Technology and Exploring Engineering, Vol.3, Issue-2, July 2013.

[14]. Vinayak D. Shinde, Vijay M. Mane, “ Pattern Recognition using Multilevel Wavelet Transform”, International Journal of Computer Applications, Volume 49- No.2, July 2012.

[15]. ZhongliangLuo, "Iris Feature Extraction and Recognition Based on Wavelet-Based Contourlet Transform", Procedia Engineering, Elsevier, 2012.

[16]. Xuezhi Wen, Wei Liu, Nan Wang, Huai Yuan, Hong Zhao, "Improved Wavelet Feature Extraction Methods Based on HSV Space for Vehicle Detection”, IAPR Conference on Machine Vision Applications, May 16-18, 2007.

[17]. Yanxia Wang, QiuqiRuan "Dual-Tree Complex Wavelet Transform based Local Binary Pattern Weighted Histogram method for palmprint recognition", Computing and Informatics, Vol. 28, 2009.

[18]. KotherMohideen, ArumugaPerumal, Krishnan and Mohamed Sathik, "Image Denoising And Enhancement Using Multiwavelet With Hard Threshold In Digital Mammographic Images”, International Arab Journal of e-Technology, Vol. 2, No. 1, January 2011.

[19]. Mathieu Lamard, Guy Cazuguel, Gw_enoleQuellec, Lynda Bekri, Christian Roux, "Content Based Image Retrieval based on Wavelet Transform coefficients distribution”, Conference proceedings : Annual International Conference of the IEEE Engineering in Medicine and Biology Society., IEEE, 2007.

[20]. Hamid Soltanian-Zadeha, FarshidRafiee-Rad, SiamakPourabdollah-Nejad D, "Comparison of multiwavelet, wavelet, Haralick, and shape features for microcalcification classification in mammograms”, journal of Pattern Recognition, Pergamon, Elsevier, 2004. 